\title{
Transparencia, participación ciudadana y gobierno electrónico: el caso del Gobierno Local de Ciudad Juárez, México'
}

\author{
Abraham Paniagua Vázquez \\ Doctor en Ciencias Sociales (Universidad Autónoma de Ciudad Juárez) \\ Profesor en la Universidad Autónoma de Chihuahua \\ apaniagua@uach.mx \\ José Eduardo Borunda Escobedo \\ Doctor en Ciencias Sociales (El Colegio de la Frontera Norte) \\ Profesor en la Universidad Autónoma de Chihuahua \\ joseeduardoborunda@gmail.com \\ Ignacio Camargo González \\ Doctor en Ciencias Sociales (El Colegio de la Frontera Norte) \\ Profesor en la Universidad Autónoma de Chihuahua \\ icamargo@uach.mx
}

En este documento se presentan los resultados de la investigación sobre la manera en la que la ciudadanía interactúa con la lógica de la transparencia y el acceso a la información relacionada a Ciudad Juárez, y a su vez se analiza como las iniciativas ciudadanas evalúan esta política del gobierno local. El aparato crítico se basa en las diferentes corrientes teóricas relacionadas con el gobierno electrónico, la gobernabilidad, la gobernanza, la participación ciudadana, la transparencia y el acceso a la información. La pregunta de investigación del presente avance es ¿Cómo se ha comportado la participación ciudadana con relación a la política de Transparencia y Acceso a la Información Pública en el Municipio de Juárez en el período 2007-2011?

Palabras-clave: transparencia y acceso a la información pública, gobierno electrónico, participación ciudadana, democracia, Ciudad Juárez.

\section{Introducción}

Ce ha vuelto común afirmar que como parte de las estrategias $\checkmark$ que proyectan nuevas relaciones entre gobernantes y gobernados, especialmente lo referente a la transparencia y rendición de cuentas, el que se hayan adoptado en México políticas públicas de gobierno electrónico.

Bajo la lógica de convertir a la administración pública en un ente más eficiente, eficaz y con mayor acceso a la ciudadanía, a partir del gobierno encabezado por el presidente Ernesto Zedillo Ponce de León y especialmente durante la gestión de Vicente Fox Quezada el aparato

1. Este trabajo forma parte del convenio de colaboración entre el Gobierno Municipal 20102013 de Juárez y el Cuerpo Académico UACH-CA 120 "Política, Gobierno y Sociedad" adscrito a la Universidad Autónoma de Chihuahua, Facultad de Ciencias Políticas y Sociales. 
burocrático mexicano ha desarrollado alternativas de gobierno basadas en la operación intensiva de las Tecnologías de la Información y Comunicación (TICS). Esta acción de gobierno se vio respaldada por la aprobación del día 11 de junio del 2002 de la Ley Federal de Acceso a la Información Pública Gubernamental (LTAIP), y en el caso de Ciudad Juárez, por la Ley de Transparencia y Acceso a la Información Pública del Estado de Chihuahua LTAIPECH del 10 de octubre del 2005. Ambos marcos jurídicos normaron la institucionalidad del gobierno electrónico e impulsaron la interacción entre dependencias públicas y ciudadanos mediante el uso de las Tics, especialmente el Internet.

La emergencia de la transparencia y la rendición de cuentas como parte de la cotidianidad política de la sociedad ha dado pie a varias vertientes de investigación como: la evaluación al gobierno electrónico; el impacto en la modernización de la administración pública; su relación con la gobernabilidad y gobernanza; el acceso a la información como elemento central de la consolidación democrática; la promoción al interés público por parte del gobierno; y, las presiones globales para generar las condiciones sobre el acceso a la información.

A la par de estos intereses académicos, el nuestro persigue el objetivo de discutir sobre la participación ciudadana y su relación con la política de transparencia y acceso a la información pública en el Municipio de Juárez, México.

El planteamiento de este objetivo permite distinguir que la participación ciudadana se presenta en dos vertientes: una relación vertical, en la cuál la ciudadanía se limita a requerir información de alguna de las distintas funciones de lo público; y una relación horizontal, en la que la función ciudadana no se limita sólo a preguntar, además evalúa la tarea del gobierno local de incentivar la práctica de la transparencia y el acceso a la información.

La pregunta general sobre la cuál esta investigación ahonda es: ¿Cómo se ha comportado la participación ciudadana con relación a la política de Transparencia y Acceso a la Información Pública en el Municipio de Juárez en el período 2007-2011?

A partir de la cuál se generan dos interrogantes: ¿Cuáles son las dimensiones en las que la participación ciudadana puede afectar la política de transparencia y acceso a la información pública del gobierno local? ¿Qué ventajas o beneficios genera la participación ciudadana sobre transparencia y acceso a la información al gobierno municipal?
La idea principal que guía este trabajo es que la participación ciudadana configura la política de transparencia y acceso a la información. Es decir, la ciudadanía se erige como actor activo, ya que genera las capacidades necesarias no sólo para requisitar información, además evalúa el papel del gobierno local en esta política pública.

Para perseguir nuestros objetivos y dar respuesta a nuestras preguntas, en esta propuesta se analizó información divulgada de manera electrónica por el Instituto Juarense para la Transparencia y Acceso a la Información Pública, la instancia señalada como responsable por la Ley de Transparencia y Acceso a la Información Pública del Estado de Chihuahua de garantizar que los sujetos obligados cumplan la LTAIPECH; y en información proporcionada por Ciudadanos por Municipios Transparentes (CIMTRA) y por Ciudadanos por una Mejor Administración Pública (CIMAP), iniciativas ciudadanas, que entre otros objetivos evalúan la transparencia en los gobiernos locales e incentivan la participación social en la búsqueda de mejores prácticas gubernamentales que incidan en el desarrollo social.

Este trabajo se estructura en tres epígrafes: en el primero se presentan las aproximaciones básicas sobre los conceptos que explican la participación ciudadana mediante la transparencia y el acceso a la información; en el segundo se analiza la información consultada ex profeso; mientras que el tercer epígrafe se presenta a modo de conclusiones e implicaciones. La estructura se complementa con la bibliografía.

\section{Transparencia, rendición de cuentas, participación ciudadana y gobierno electrónico. Aproximaciones conceptuales}

Doctrinarios de la democracia ${ }^{2}$ y formas de gobierno resultado de ella afirman que tanto los asuntos de interés general como las acciones de quienes los administran deben sujetarse a escrutinio público. Así, la práctica de este escrutinio reflejado en la transparencia y rendición de cuentas permite que los ciudadanos vigilen el óptimo desempeño del aparato burocrático, dotando de esta manera, de legitimidad y credibilidad al régimen político, mientras que la ciudadanía va adquiriendo madurez política e

2. La transparencia y la rendición de cuentas tienen fundamentos democráticos eminentes, su propuesta también sirve para combatir la corrupción. En estos términos, las implicaciones de los actos corruptos impacta negativamente en la credibilidad de las instituciones, en el desarrollo económico de la sociedad y en general, sobre las condiciones de vida de la población (Reyes Heroles, 2004). A pesar de su inminente importancia, en este trabajo abordamos a la corrupción como elemento marginal. 
interés por la correcta marcha de su gobierno, evitando la cultura de la opacidad, propia de sociedades en las que se niega la presencia de ciudadanos libres $^{3}$ (Uvalle, 2008; Emmerich, 2004; O’Donnell, 1998; Prud'Homme, 1997).

Alejandro Ferreiro (1999) sostiene que la utilidad de la transparencia en un gobierno democrático es necesaria ya que: sanciona e inhibe la comisión de actos ilícitos; es propia medida de la conducta ética; $\mathrm{y}$, sirve de regulación social. Al respecto, Mauricio Merino (2005, p. 17) afirma que:

La transparencia sirve como un instrumento y como unincentivo para recordarles a los funcionarios públicos que sus funciones solamente se justifican en la medida en que se correspondan con el interés público, que se atengan a las reglas del juego públicamente fijadas, y que rindan cuentas de las decisiones que toman a nombre de todos.

Sobre sus modalidades, Ferreiro (1999) reconoce la transparencia activa y la pasiva. La transparencia activa se presenta cuando la administración pública difunde de manera periódica y sistematizada la información relacionada a sus actividades. Este tipo de transparencia se genera en la obligación que tienen ciertas dependencias estatales para que, con la periodicidad estipulada en la norma, hagan pública información necesaria para que la sociedad evalúe sus actividades. La transparencia pasiva refleja la obligación estatal para facilitar a los ciudadanos que lo soliciten el acceso a información que obre en poder de dependencias gubernamentales, excepto aquella información catalogada como secreta o reservada, tal como la relativa a la seguridad nacional, la que garantice salvaguardar la condición de uno o más individuos, o de importancia estratégica para la sobrevivencia del Estado.

En el mismo tono, Ricardo Uvalle (2008) afirma para los gobernados, la ventaja institucional de conocer cuándo, dónde, por qué y cómo las autoridades actúan para dar vida a los procesos de gobierno, la proporciona la transparencia. En general, la práctica de la transparencia es condición necesaria para la sobrevivencia de la democracia, ya que permite el acceso ciudadano oportuno a la información relativa al desempeño gubernamental (López-Ayllón, 2005).

Como corolario, Gustavo Ernesto Emmerich (2004) afirma que las corrientes contemporáneas estudiosas de la transparencia y la rendición de cuentas enfatizan la importancia de que la ciudadanía tome parte activa, directa y efectiva en la vigilancia y la conducción de los asuntos públicos. El reto entonces para el estímulo de la convivencia democrática es generar en los ciudadanos intereses para la participación generalizada y el sentido de corresponsabilidad en los asuntos de interés público.

Visto así, la participación ciudadana requiere que el sistema político garantice la progresiva incorporación de la sociedad a los procesos de toma de decisiones, como una fehaciente prueba de que la sociedad forma parte del ente estatal. ${ }^{4}$

En correspondencia con la gobernabilidad, la gobernanza ${ }^{5}$ y el empoderamiento popular, la participación ciudadana enmarca entre otras cosas: el reconocimiento a grupos de la sociedad organizados; tener acceso a las funciones públicas; el derecho y la obligación de incidir en las políticas públicas; y, la vigilancia de la aplicación de los recursos del erario público. El reconocimiento de estos elementos supone la reivindicación de las instituciones para garantizar:

[...] la presencia de los intereses de la ciudadanía en el seno del Estado, en la definición de las políticas públicas y proyectos de desarrollo económico y social que necesariamente incorporaría al mayor número posible de la sociedad. (Montesinos, 2004, p. 98)

En México, la propuesta de hacer asequible a la ciudadanía la transparencia, el acceso a la información y por ende, la rendición de cuentas se ha venido consolidando a través del gobierno electrónico. Así, el Sistema Nacional e-México, se concibió como una posibilidad viable de sistematizar procesos gubernamentales para el beneficio de la sociedad y constituyó una respuesta pública a los desafíos y exigencias ${ }^{6}$ enmarcados en el contexto de la globalización (Sour, 2007). La conexión globalización - transparencia se

3. "Cuando el Estado controla y regula de manera contundente la información pública se convierte en un peligro para las libertades civiles y políticas de la sociedad moderna" (Uvalle, 2008, p. 111).

4. En cuanto a la intensidad de la participación de la ciudadanía en el ámbito público, el filósofo Norberto Bobbio (1989) sugiere que tanto el exceso de la misma como su ausencia generan crisis en la toma de decisiones desde la esfera pública, ya que en el primer caso los ciudadanos cuestionan a los gobernantes y los superan debido a la fuerte participación; mientras que la ausencia de participación política genera falta de credibilidad en el régimen.

5. La gobernabilidad debe considerar: la construcción de consensos; el vigor de la acción pública; y, las nuevas reglas del juego. Por su parte, la gobernanza democrática valora y acepta la existencia de redes sociales y políticas que reclaman su derecho a participar e intervenir en la operación directa del Estado, destacando su autonomía frente a los poderes e instituciones del propio Estado. La gobernanza se vincula con modos específicos del poder, destaca la importancia de que los asuntos públicos no sean monopolio estatal (Uvalle, 2007, p. 53-63).

6. Hacia en interior del aparato burocrático, las presiones globales corresponden a exigencias por la modernización, así, las propuestas de acuerdos internacionales, la aplicación de las Tecnologías de la Información y Comunicación (TICS) a los procesos burocráticos, y las reformas a la administración pública se hicieron presentes en el México de inicios del siglo XX. 
explica ya que las exigencias globales pueden generar cambios al interior del comportamiento de las administraciones públicas, incluyendo la apertura a la transparencia (Scott, 1998).

Luego entonces, el foro de interacción virtual generado por las Tics, donde ciudadanos, organizaciones de la sociedad civil, empresas y gobierno disciernen sobre asuntos públicos y sociales constituye el leitmotif del gobierno electrónico, que es definido como:

[...] una forma de organización del Estado que integra las interacciones e interrelaciones entre el Estado y los ciudadanos, industrias privadas, clientes e instituciones públicas a través de lautilización de tecnologías de la información y comunicación modernas. (Schedler et. al., 2004, p. 5)

Cabe señalar que la posibilidad de consolidar foros de participación ciudadana que reclamen mayor acceso a la información de interés general utilizando tecnología avanzada no genera per se resultados de transparencia ni demandas hacia los gobiernos. Bhatnagar Subhash $(2003$, p. 2) sugiere que para incrementar la rendición de cuentas en aras de reducir la corrupción, el proceso de apertura mediante el uso de las Tics debe inducir una secuencia de objetivos: asegurarse del incremento en el acceso a la información; claridad en los marcos jurídico - legales; y, la construcción de un marco normativo que garantice el seguimiento de las acciones y decisiones de los servidores públicos.

Bajo esta lógica, el gobierno electrónico generará influencia en asuntos de transparencia y rendición de cuentas, ampliando el acceso a la información, mejorando la estructura de la administración pública y sobre todo, generando las condiciones para que se presente el debate entre los distintos sectores interesados en el quehacer público.

\section{Entre la gobernabilidad y la gobernanza. La participación ciudadana en la transparencia y el acceso a la información}

El juego dialéctico entre democracia - transparencia y gobernabilidad - gobernanza genera un lugar común: el espectro multicéntrico de actores interesados en acceder al poder de la toma de decisiones de los asuntos públicos, escenario reflejado en "la cabida de nuevos actores y movimientos que reflejan la pluralidad del espacio público" (Uvalle, 2008, p. 100). En este ejercicio de empoderamiento social emergen como actores de la política organizaciones de la sociedad civil, colectivos, movimientos urbanos y líderes sociales que hasta antes de esta apertura democrática, es decir durante la democracia representativa, sólo influían de manera indirecta y esporádica, especialmente durante el proceso electoral (Peters, 2005).

En esta transición a la democracia participativa, es necesaria una distinción entre dos elementos que intentan el empoderamiento de la sociedad: la gobernabilidad y la gobernanza. Ambos aluden a la

Diagrama 1 - Democracia, transparencia, gobernabilidad y gobernanza.

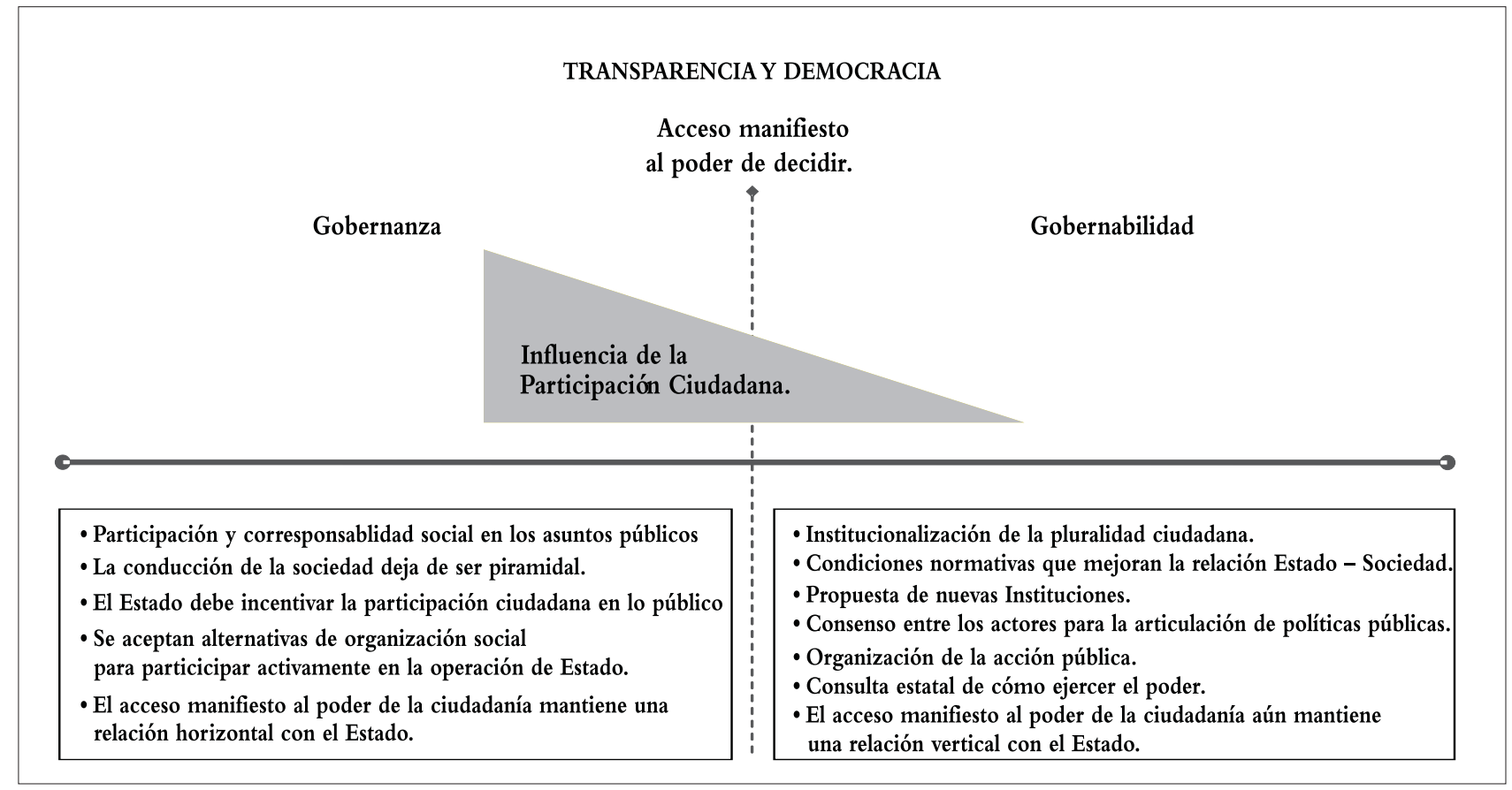

Fuente: Elaboración propia con base en Uvalle, 2008; Peters, 2005; Emmerich, 2004. 
interacción Estado - sociedad; el primero relativa a la permisividad de la existencia institucional de los contrarios, al respeto de la pluralidad y la diferencia, al consenso garante de cubrir las expectativas de desarrollo y desempeño social (Uvalle, 2008); mientras que la lógica que subyace en la visión de la gobernanza es que a mayor participación ciudadana, mayor calidad en las tareas de gobernar. En la percepción de la gobernanza se presenta como fundamental la influencia de la sociedad en las políticas destinadas a su propio desarrollo (Peters, 2005).

Si bien tanto la gobernabilidad como la gobernanza mantienen en su racionalidad legitimar al gobierno mediante la participación ciudadana, el punto de diferencia entre ambos conceptos es pragmático: acceder de manera manifiesta al poder de decidir, pasar del reconocimiento oficial de las demandas de inclusión social a la toma de decisiones. Mientras que la gobernabilidad se entiende como “... la condición insoslayable para llevar a cabo la articulación de actores, poderes, organizaciones e intereses que requieren viabilidad para desarrollarse como fuerzas que ocupan un lugar en la estructura básica de la sociedad" (Uvalle, 2008, p. 101), la gobernanza "representa un intento por involucrar más a la sociedad en la tarea de gobernar y por reducir los elementos jerárquicos del sistema" (Peters, 2005, p. 590). Un concepto mantiene relación vertical con el gobierno, el otro ejerce una relación horizontal.

Ya hemos mencionado que el principal reto en materia de convivencia democrática es alentar la participación ciudadana en los asuntos públicos, construyendo espacios de intervención novedosos que garanticen alternativas de gobernar. En este sentido, el actuar ciudadano en el ámbito de la evaluación es síntoma de la gestión a favor del interés general. El trabajo desarrollado por Ciudadanos por Municipios Transparentes CIMTRA y por Ciudadanos por una Mejor Administración Pública Cimap ejemplifica esta propuesta.

CIMTRA Ciudadanos por Municipios Transparentes, es un colectivo de organizaciones sociales que mide la transparencia de los gobiernos locales, su accionar sigue tres principios:

a) El municipio como la autoridad más cercana a la gente, por lo tanto su influencia en la vida cotidiana es importante.

b) La promulgación de los diez Criterios de Mejores Practicas en Gobiernos Locales por la Conferencia Hábitat II ONU, celebrada en Estambul en 1996, de donde se desprende la promoción de la responsabilidad y la Transparencia.

c) Disminuir la brecha entre los municipios del país.
Los objetivos que persigue CIMTRA buscan generar un movimiento ciudadano nacional que fomente una nueva cultura a favor de ejercer el derecho de acceso a la información pública y la transparencia a fin de lograr la rendición de cuentas de los gobiernos locales; y que el ciudadano incida en la adecuación del marco normativo en la materia, así como una mayor presencia en la toma de decisiones. ${ }^{7}$

Por su parte Ciudadanos por una Mejor Administración Pública CIMAP, es una organización formada a raíz de la inquietud ciudadana interesada por mejorar sustancialmente la actuación del gobierno. $\mathrm{Su}$ racionalidad gira en torno a impulsar mecanismos para que los gobiernos mejoren su efectividad, y los juarenses tengan mejor calidad de vida. Se organizan en torno al siguiente Código de Comportamiento:

1. Primero los intereses de Juárez, buscando siempre los intereses generales por encima de los particulares o de grupo.

2. Buscar el sentir juarense. Promover una membresía participativa que refleje los intereses de la comunidad.

3. Promover la profesionalización del gobierno. Proponiendo modelos prácticos, creativos y probados.

4. Practicar la cortesía en la comunicación.

5. Ejercer la crítica al gobierno sin distinción de partidos o ideología política.

6. Independencia. No recibimos recursos del gobierno, las opiniones vertidas son exclusivamente a título personal y no representan las posiciones de las organizaciones a las cuales podríamos pertenecer.

7. Hacer públicas las propuestas emanadas al interior de CIMAP. Aceptar y avalar las decisiones que tome la mayoría.

8. Fortalecer al CIMAP, hacer sólo uso de la información interna que haya sido oficialmente divulgada por el grupo.

CIMTRA, a través de CIMAP mide y evalúa la transparencia en el gobierno local en tres campos o áreas: a. información ciudadana; b. atención ciudadana; y, c. espacios de comunicación gobierno-ciudadano. Estos tres campos o áreas pretender responder a las preguntas: a. ¿que se informa al ciudadano? b. ¿cómo se atiende al ciudadano? y c. ¿cómo participan los ciudadanos junto con el gobierno?

El escrutinio conlleva la aplicación de un cuestionario integrado por 31 indicadores, del resultado se integra una lista de los municipios evaluados que siguen el orden según la calificación asignada (http:// www.cimap.org.mx/). En el caso del gobierno municipal de Ciudad Juárez las evaluaciones han producido los siguientes resultados: 
Cuadro 1 - Clasificación del Municipio de Juárez de acuerdo a la Evaluación de la Transparencia Municipal de CIMTRA - CIMAP.

\begin{tabular}{|c|c|c|c|}
\hline Año & Mes & Lugar Nacional & Calificación \\
\hline 2011 & Mayo & 3 & 74.5 \\
\hline 2010 & Diciembre & 3 & 69.0 \\
\hline 2010 & Abril & 7 & 53.3 \\
\hline 2009 & Octubre & 9 & 55.4 \\
\hline 2009 & Mayo & 10 & 47.6 \\
\hline
\end{tabular}

Fuente: Elaboración propia con datos de CIMTRA - CIMAP.

El desarrollo de las evaluaciones sobre transparencia y acceso a la información que elaboran CIMTRA -CIMAP evidencia una evolución en el caso del Municipio de Juárez, ya que en cada período de escrutinio muestra un constante avance. De esta manera ha pasado de ocupar el escaño número 10 en mayo del 2009, a mantenerse en la tercera posición en las últimas evaluaciones de diciembre del 2010 y mayo del 2011, por encima de municipios importantes como Chihuahua, Tlaxcala, Tlaquepaque, Puerto Vallarta o Guadalajara.

Para el gobierno local sobresalir en una clasificación de transparencia a nivel nacional representa el reconocimiento ciudadano de que, por lo menos en este rubro, existe una estrategia definida para consolidar elementos de gobierno participativo, nada mal para una administración pública de una ciudad que en los últimos tres años ha sido fuertemente golpeada por la violencia, la inseguridad pública y el desempleo.

El cuadro 2 muestra hasta la descripción del "bloque referente" la evolución del Municipio de Juárez en las cinco evaluaciones hasta ahora realizadas. Los aspectos que se examinan son tres: información a la ciudadanía; espacios de comunicación; y, atención a la ciudadanía.

Los bloques referentes que contiene el aspecto de información a la ciudadanía son: gastos; obras; bienes y sus usos; administración; y, urbanismo. Este bloque contiene indicadores tan diversos como: información de proveedores; gastos por asesorías; gastos de representación o viáticos de funcionarios de primer nivel; contratos de obras y servicios por asignación directa; propuesta de obra del COPLADEMUN; bienes inmuebles patrimoniales propiedad del gobierno; detalles de los programas sociales del Municipio; listado de indicadores y resultados; y, cambios de uso de suelo, entre otros.

El aspecto espacios de comunicación contiene los bloques referentes de: consejos; participación ciudadana; y, cabildo. Manejan los indicadores de sesiones ordinarias públicas; existencia de reglamento de participación ciudadana; sesiones de cabildo ordinarias públicas y abiertas; convocatoria pública para seleccionar integrantes ciudadanos; y, organismo ciudadano para revisar, supervisar y evaluar ejecución de obra pública y de programas sociales, entre otros.

Atención a la ciudadanía es el tercer aspecto que se evalúa, en este aspecto se incluyen los bloques referentes de: información de servicios; mecanismos de atención ciudadana; y, oficina, módulo o mecanismo de acceso a la información pública.

El comportamiento ascendente sostenido por el Municipio de Juárez en la escala de evaluación sugiere que la transparencia y el acceso a la información pública son elementos asequibles para la ciudadanía interesada, sin embargo, la evaluación presentada por CIMTRA - CIMAP no permite vislumbrar elementos de una participación ciudadana activa en la toma de decisiones y en el acceso al poder. De cualquier manera este ejercicio ciudadano representa una figura política que genera legitimidad a los gobiernos examinados y se incorpora al marco de las democracias participativas y la gobernabilidad.

Otro de los elementos que reflejan participación ciudadana en aras de la transparencia y el acceso a la información son las solicitudes relacionadas con la ad-

Cuadro 2 - Criterios para la Evaluación de la Transparencia Municipal CIMTRA - CIMAP, caso de Ciudad Juárez.

\begin{tabular}{|c|c|c|c|c|c|c|}
\hline Aspecto & $\begin{array}{l}\text { Bloque } \\
\text { Referente }\end{array}$ & $\begin{array}{c}\text { Mayo } \\
2009 \\
\end{array}$ & $\begin{array}{c}\text { Octubre } \\
2009\end{array}$ & $\begin{array}{l}\text { Abril } \\
2010\end{array}$ & $\begin{array}{c}\text { Diciembre } \\
2010\end{array}$ & $\begin{array}{c}\text { Mayo } \\
2011 \\
\end{array}$ \\
\hline \multirow{5}{*}{$\begin{array}{l}\text { Información a la } \\
\text { Ciudadania }\end{array}$} & Gastos & $32.6 \%$ & $52.4 \%$ & $42.9 \%$ & $57.10 \%$ & $71.43 \%$ \\
\hline & Obras & $28.6 \%$ & $27.8 \%$ & $27.8 \%$ & $22.20 \%$ & $66.67 \%$ \\
\hline & Bienes e sus usos & $29.4 \%$ & $29.4 \%$ & $23.5 \%$ & $41.20 \%$ & $47.06 \%$ \\
\hline & Administración & $50.0 \%$ & $54.8 \%$ & $51.6 \%$ & $54.80 \%$ & $80.65 \%$ \\
\hline & Urbanismo & $0.0 \%$ & $60.0 \%$ & $60.0 \%$ & $80.0 \%$ & $100.00 \%$ \\
\hline \multirow{3}{*}{$\begin{array}{l}\text { Espacios de } \\
\text { Comunicación }\end{array}$} & Consejos & $28.6 \%$ & $14.3 \%$ & $14.3 \%$ & $85.70 \%$ & $14.29 \%$ \\
\hline & Participación Ciudadana & $60.00 \%$ & $60.00 \%$ & $60.00 \%$ & $80.00 \%$ & $90.00 \%$ \\
\hline & Cabildo & $100.00 \%$ & $100.00 \%$ & $100.00 \%$ & $100.00 \%$ & $100.00 \%$ \\
\hline $\begin{array}{l}\text { Atención a la } \\
\text { Ciudadania }\end{array}$ & Atención Ciudadana & $100.00 \%$ & $100.00 \%$ & $100.00 \%$ & $100.00 \%$ & $100.00 \%$ \\
\hline & & $47.0 \%$ & $55.4 \%$ & $53.3 \%$ & $69.00 \%$ & $74.50 \%$ \\
\hline
\end{tabular}


Cuadro 3 - Estadísticas de Transparencia 2007 - 2011 en el Estado de Chihuahua.

\begin{tabular}{|c|c|c|c|c|c|c|}
\hline & \multicolumn{3}{|c|}{$\begin{array}{c}\text { Sujetos obligados con mayor número } \\
\text { de solicitudes en el Estado }\end{array}$} & \multicolumn{3}{c|}{$\begin{array}{c}\text { Ayuntamientos del Estado } \\
\text { más requeridos }\end{array}$} \\
\hline Año & Lugar & Sujeto & Cantidad & Lugar & Ayuntamiento & Cantiadad \\
\hline 2007 & 1 & Ayuntamiento de Juárez & 284 & 1 & Juárez & 284 \\
\hline 2008 & 1 & Ayuntamiento de Juárez & 358 & 1 & Juárez & 358 \\
\hline 2009 & 1 & Ayuntamiento de Juárez & 310 & 1 & Juárez & 310 \\
\hline 2010 & 1 & Ayuntamiento de Juárez & 491 & 1 & Juárez & 491 \\
\hline $2011 \star$ & 2 & Ayuntamiento de Juárez & 151 & 1 & Juárez & 151 \\
\hline
\end{tabular}

Fuente: Elaboración propia con información del Instituto Chihuahuense para la Transparencia y Acceso a la Información Publica (ICHITAIP) y Sistema INFOMEX. ^Estadística hasta el 31 de marzo de 2011.

ministración del Municipio de Juárez. El cuadro 3 refleja que el Ayuntamiento de Juárez ha sido del 2007 al 2010 el sujeto obligado en el Estado de Chihuahua más cuestionado, por encima de organismos públicos como el Supremo Tribunal de Justicia, la Universidad Autónoma de Chihuahua, la Universidad Autónoma de Ciudad Juárez y todas las dependencias de Gobierno del Estado. Hasta el 31 de marzo del 2011 sólo el Instituto Chihuahuense de Transparencia y Acceso a la Información Pública ICHITAIP generaba más solicitudes de información. Por la el rubro de Ayuntamientos, el de Juárez ha sido el que más preguntas generó desde el 2007 hasta el 31 de marzo del 2011.

Las causas son variadas si consideramos que la ciudad cuenta con el 39.04\% del total de la población del Estado, o bien, las dudas ciudadanas que generaron la puesta en marcha de proyectos públicos controvertidos y de escasos o nulos resultados como el fallido transporte semimasivo, el crecimiento sin sustento de la fuerza policiaca municipal y la colaboración municipal con las fuerzas federales por militarizar la ciudad.

Lo trascendental de las estadísticas estatales sobre transparencia es que muestran a una ciudadanía inquieta, critica, interesada en participar en los asuntos públicos.

\section{Conclusiones e implicaciones}

Las políticas públicas sobre transparencia y acceso a la información que se basan en el uso de las Tics encuadran en las exigencias ciudadanas para abrir el círculo de lo público al escrutinio y crítica social que impactan de alguna manera en las tareas de gobernar.

En este contexto, en el caso de Ciudad Juárez se localizan prácticas ciudadanas llevadas a cabo por individuos u organizaciones sociales, que utilizando tecnologías de la información TICS y a nombre de la transparencia y el acceso a la información pública desarrollan acciones dignas de un escenario democrático caracterizado por la formación de gobiernos participativos.
Podemos afirmar que la participación ciudadana legitima a la administración pública local en cuanto a su relación con sus gobernados, a la capacidad para procesar información solicitada, y en un sentido restringido, en el ejercicio de los recursos, que en conjunto permiten el desarrollo de la gobernabilidad local. A la vez, el acceso a la información pública por parte de la ciudadanía reivindica a los gobiernos como abiertos, democráticos y comprometidos con la eficacia y el buen desempeño.

Sin embargo, a pesar de estos innegables avances, la participación ciudadana relacionada a la transparencia y acceso a la información pública no alcanza aún fortalecer los elementos básicos de la gobernanza. Sus motivos son varios, del lado de la sociedad se requiere de una participación más amplia y enfocada no sólo a la solicitud de información y a la evaluación del accionar público sobre la temática, se necesita una sociedad que dé seguimiento a las observaciones generadas en sus evaluaciones mediante la movilización de recursos y la consolidación de sus relaciones con aliados clave, que en conjunto le permitan formar parte activa del gobierno. Al respecto, un par de preguntas pueden ayudar: ¿que hace el gobierno con las observaciones que se generan? ¿cuál es la naturaleza de la diversidad de actores que, de manera coordinada evalúan y observan al gobierno? ¿el movimiento generado por organizaciones de la sociedad civil es realmente incluyente? El objetivo de estos cuestionamientos es conocer hasta que punto la participación ciudadana, la transparencia y acceso a la información impactan en la inclusión de las exigencias sociales en la forma de ejercer el poder.

Por su parte, el gobierno local debe contemplar que con la respuesta a las solicitudes de información, si bien se cumple una parte de su obligación, no se abarca la idea básica de revertir estructuras jerárquicas, ni se apertura el sector público a influencias sociales. Lo que subyace a la idea de gobernanza es que tanto el gobierno como actores y organizaciones sociales estrechen vínculos con la intención de que las propuestas de desarrollo generadas en el seno del gobierno sean contundentes en las decisiones en asuntos de interés general de vertical a horizontal. 
Para el caso del gobierno local de Ciudad Juárez, los avances presentados en la evolución de las prácticas de transparencia y acceso a la información pública relacionados al $e$-gobierno no deben ser vistos como

\section{Referências}

BOBBIO, Norberto. Estado, gobierno y sociedad. México: FCE, 2006.

DE SARRATUD, Alida. El verdadero significado del protagonismo de la participación ciudadana en la gestión pública. Observatorio Laboral Revista Venezolana, v. 3, n 5, enero/junio, p. 61-74, 2010.

EMMERICH, Gustavo Ernesto. Transparencia, rendición de cuentas, responsabilidad gubernamental y participación ciudadana. Polis: Investigación y Análisis Sociopolítico y Psicosocial, año/v. 2, nº 4, segundo semestre, p. 67-90, 2004. FERREIRO, Alejandro. Dinero, política y transparencia: el imperativo democrático de combatir la corrupción, ponencia. Presentada en la Novena Conferencia AntiCorrupción. Durban, Sudáfrica, 1999.

LÓPEZ-AYLLÓN, Sergio. La creación de la Ley de Acceso a la Información en México: Una perspectiva desde el Ejecutivo Federal. En: CANTÚ, Hugo Concha; LÓPEZ-AYLLÓN, Sergio y TACHER, Lucy (Coord.). Transparentar al Estado: la experiencia mexicana de acceso a la información. Instituto de Investigaciones Jurídicas, UNAM, México, 2005.

MERINO, Mauricio (Coord.). Transparencia: libros, autores, ideas. CIDE-IFAI, México, 2005.

MONTESINOS, Rafael. La participación ciudadana en la "modernidad democrática" mexicana. El Cotidiano, año/v. 20, no 128, p. 93-104, noviembre/diciembre, 2004.

O' DONELL, Guillermo. Accountability horizontal. En Estudios Políticos, n 19, 1998.

PETERS, Guy. Gobernanza y burocracia pública: ¿Nuevas formas de democracia o nuevas formas de control? Foro Internacional, v. XLV, $\mathrm{n}^{\circ}$ 4, 2005.

PRUD'HOMME, Jean. Consulta popular y democracia directa. IFE, México, 1997. el objetivo final, ese debe ser el utilizar el performance democrático y de participación ciudadana para mejorar la rendición de cuentas y para incorporar el sentir ciudadano a la toma de decisiones.

REYES HEROLES, Federico. Corrupción: de los ángeles a los índices. IFAI, México, 2004.

RODRÍGUEZ, Gladys. Gobierno electrónico: Hacia la modernización y transferencia de la gestión pública. Revista de Derecho, no 21, p. 1-23, 2004.

SCOTT, Richard. Organizations: rational, natural and open systems. Prentice Hall, Englewood Cliffs, 1998.

SCHELDER, Kuno et. al. Managing the electronic government: from vision to practice. Greenwich: Information Age Publishing, 2004.

SHEDLER, Andreas. ¿Qué es la rendición de cuentas? IFAI, México, 2004.

SOUR, Laura. Evaluando al gobierno electrónico: avances en la transparencia de las finanzas públicas estatales. Economía, Sociedad y Territorio, año/v. VI, no 23, enero/ abril, p. 613-654, 2007.

SUBHASH, Bhatnagar. Administrative corruption: How does e-government help? 2003. Documento en línea $<$ www.worldbank.org/publicsector/egov/corruption. doc $>$. Acceso el: 28 de mayo 2011.

UVALLE, Ricardo. Gobernabilidad, transparencia y reconstrucción del Estado. Revista Mexicana de Ciencias Políticas y Sociales, v. L, no 203, mayo/agosto, p. 97-116, 2008.

\section{Páginas en Internet}

Disponible en: <http://www.cimtra.org.mx>. Acceso el: 1 y 2 de junio del 2011.

Disponible en: <http://www.cimap.org.mx>. Acceso el: 26 y 27 de mayo del 2011. 


\title{
Transparência, participação cidadã e governo eletrônico: o caso do
}

\section{governo municipal de Ciudad Juárez, México}

\section{Resumo}

O texto apresenta o resultado de um estudo sobre a maneira na qual a cidadania interage com a lógica da transparencia e do acesso à informação na Cidade Juárez, analisando como as iniciativas de cidadania avaliam esta política do governo local. A referência teórica do texto está baseada nas diferentes correntes que estudam o governo eletrônico, a governabilidade, a governança, a participação cidadã, a transparência e o acesso à informação. A questão principal deste artigo é: Como tem se comportado a participação cidadã com relação à política de transparência e acesso à informação pública no municipio de Juaréz no período de 2007-2011?

Palavras-chave: transparência e acesso à informação pública, governo eletrônico, participação cidadã, democracia, Ciudad Juárez.

\section{Transparency, citizen participation and e-government: the case of the Local Government of Ciudad Juárez, Mexico}

\begin{abstract}
This text presents the results of a study on the way in which citizens interact with the transparency logic and access to information in Ciudad Juárez, analyzing how citizen's initiatives evaluate the local government policy; based on different theoretical trends related to e-government, governance, citizen participation, transparency and access to information. The research question is related to how citizen's participation has behaved in relation to transparency policy and access to public information in Ciudad Juárez from 2007 to 2011.
\end{abstract}

Key words: transparency and access to public information, e-government, citizenship, democracy, Ciudad Juárez.

Data de recebimento do artigo: 10/09/2011

Data de aprovação do artigo: 29/01/2012 